\title{
Criminologie
}

\section{Au-delà de la criminalisation : l'immigration et les enjeux pour la criminologie}

\section{João Velloso}

Volume 46, numéro 1, printemps 2013

La criminalisation de l'immigration

URI : https://id.erudit.org/iderudit/1015293ar

DOI : https://doi.org/10.7202/1015293ar

Aller au sommaire du numéro

\section{Éditeur(s)}

Les Presses de l’Université de Montréal

ISSN

0316-0041 (imprimé)

1492-1367 (numérique)

Découvrir la revue

Citer cet article

Velloso, J. (2013). Au-delà de la criminalisation : l'immigration et les enjeux pour la criminologie. Criminologie, 46(1), 55-82.

https://doi.org/10.7202/1015293ar
Résumé de l'article

Le but de cet article est de discuter de l'importance croissante des punitions administratives dans le champ pénal, à partir de la judiciarisation des conflits d'immigration au Canada. À l'aide d'une analyse documentaire et des résultats d'une enquête de terrain menée à la Commission de l'immigration et du statut de réfugié du Canada entre 2007 et 2009, nous présenterons certaines caractéristiques de la mise en forme des litiges en droit de l'immigration et de leur façon de punir et nous soutiendrons que celles-ci diffèrent substantiellement de celles propres à la mise en forme pénale. Notre objectif ultime consistera à problématiser l'idée de criminalisation de l'immigration comme une catégorie capable de nuancer la complexité des formes de réaction sociale administratives. Nous suggérerons qu'il faut plutôt appréhender la punition en droit administratif comme telle (mesures de police et sanctions administratives) et repenser son rôle au sein du champ pénal, et ce, afin de mieux comprendre l'ensemble des réactions sociales dans les différentes institutions juridico-politiques, l'interaction et la complémentarité de celles-ci ainsi que leurs logiques de gouvernance, de mise en forme et leurs implications sociales.
Ce document est protégé par la loi sur le droit d'auteur. L'utilisation des services d’Érudit (y compris la reproduction) est assujettie à sa politique d'utilisation que vous pouvez consulter en ligne.

https://apropos.erudit.org/fr/usagers/politique-dutilisation/ 


\title{
Au-delà de la criminalisation: l'immigration et les enjeux pour la criminologie
}

\author{
João Velloso \\ Candidat au doctorat en criminologie \\ Professeur à la Faculté des sciences sociales \\ Université d'Ottawa \\ joao.velloso@uottawa.ca
}

\begin{abstract}
RÉSUMÉ - Le but de cet article est de discuter de l'importance croissante des punitions administratives dans le champ pénal, à partir de la judiciarisation des conflits d'immigration au Canada. À l'aide d'une analyse documentaire et des résultats d'une enquête de terrain menée à la Commission de l'immigration et du statut de réfugié du Canada entre 2007 et 2009, nous présenterons certaines caractéristiques de la mise en forme des litiges en droit de l'immigration et de leur façon de punir et nous soutiendrons que celles-ci diffèrent substantiellement de celles propres à la mise en forme pénale. Notre objectif ultime consistera à problématiser l'idée de criminalisation de l'immigration comme une catégorie capable de nuancer la complexité des formes de réaction sociale administratives. Nous suggérerons qu'il faut plutôt appréhender la punition en droit administratif comme telle (mesures de police et sanctions administratives) et repenser son rôle au sein du champ pénal, et ce, afin de mieux comprendre l'ensemble des réactions sociales dans les différentes institutions juridico-politiques, l'interaction et la complémentarité de celles-ci ainsi que leurs logiques de gouvernance, de mise en forme et leurs implications sociales.
\end{abstract}

MOTS-CLÉs - Contrôle de l'immigration, punition, pénologie, politique pénale, Commission de l'immigration et du statut de réfugié du Canada (CISR).

\section{Introduction}

Durant la dernière décennie, différentes formes de régulation ont vu le jour en réponse aux attaques du 11 septembre 2001, particulièrement en matière de sécurité. Dans un discours tenu juste après les événements, l'ancien vice-président américain Dick Cheney parlera d'une «nouvelle

Traduit de l'anglais par Rabia Mzouji

Criminologie, vol. 46, $\mathrm{n}^{\circ} 1$ (2013) 
normalité $»^{1}$. Or les mesures prises sont surtout venues aggraver la situation des étrangers déjà confrontés, tout au long des années 1990, au contrôle accru des frontières des pays développés ainsi qu'à ses effets d'exclusion (Bigo, 1998; Simon, 1998; Wacquant, 1999; Calavita, 2003). Alors que ces immigrants comptaient parmi les populations les plus vulnérables (Calavita, 1998; Simon, 1998; Pratt, 2005), les nouvelles politiques mises en place sont venues brutalement détériorer leurs droits déjà limités, et ce, tant aux États-Unis que partout ailleurs en Occident. Le Canada n'y a pas échappé non plus ${ }^{2}$, même s'il faut se rappeler que le principal texte de loi régulant son immigration, la Loi sur l'immigration et la protection des réfugiés (LIPR, 2001, ch. 27), a été présenté au Sénat avant le 11 septembre 2001 et qu'il a reçu la sanction royale, sans aucun amendement, deux mois après les attentats. Quoi qu'il en soit, les discours sur la nouvelle normalité ont eu un très grand impact, notamment sur les politiques d'immigration qui ont suivi, que ce soit du côté des réglementations ou de celui des pratiques, en particulier quand elles ont concerné l'application de la LIPR et le contrôle de l'immigration ${ }^{3}$.

Cherchant à décrire la nature et les effets des réglementations et autres pratiques nouvellement adoptées, les juristes et les chercheurs en sciences sociales ont presque unanimement suggéré que le contrôle de l'immigration était devenu une forme de crimmigration, à savoir une fusion entre le droit pénal (crime) et le droit de l'immigration et des réfugiés (immigration) (Miller, 2005; Stumpf, 2006; Aas, 2011). D'autres n'ont pas eu recours à ce néologisme, mais ont parlé plus communément d'une criminalisation de l'immigration (Sayad, 1998; Palidda, 1999; Wacquant, 1999; Mathieu, 2001, 2006; Miller, 2003, 2005; Bosworth, 2008; Bosworth et Guild, 2008; Di Giorgi, 2010; Zedner, 2010; Bosworth et Kaufman, 2011). De tels concepts simplifient pourtant à l'excès la régulation des litiges liés à l'immigration. Outre le fait que ces concepts les retraduisent comme «crimes», ils masquent surtout les aspects les plus troublants du contrôle de l'immigration. Ce contrôle est en effet d'abord et avant tout, sinon exclusivement, fondé sur le droit administratif. Ainsi,

1. Voir Bob Woodward, (2001, October $\left.21^{\text {th }}\right)$.

2. On trouvera un bon inventaire des mesures prises par le Canada dans Crépeau et Jimenez (2004).

3. La Loi sur l'Agence des services frontaliers du Canada (2005, ch. 38) et le Ministère de la Sécurité publique et de la Protection civile $(2005$, ch. 10) en sont de bons exemples. Ces actes législatifs ont créé la nouvelle structure de Sécurité publique Canada et ont changé le rôle que le gouvernement fédéral joue devant les tribunaux ou en appliquant la LIPR. 
la plupart des préoccupations soulevées par les juristes relèvent du manque de garanties juridiques assurées en matière de droit de l'immigration. Or une telle situation n'est précisément possible que dans la mesure où les immigrants ne sont pas «criminalisés», mais bien aux prises avec des régimes de droit administratif. Parler de criminalisation de l'immigration pose également problème à partir du moment où les immigrants sont détenus, expulsés ou encore gardés sous surveillance sans avoir bénéficié de l'ensemble des droits généralement associés au droit pénal et à l'imposition de peines ${ }^{4}$. En ce sens, nous considérons l'affirmation de Stephen Legomsky (2007: 473, notre traduction) déjà plus pertinente: «le droit en matière d'immigration a emprunté à la justice criminelle ses dispositifs de contrôle punitif, mais sans les garanties procédurales censées les accompagner».

Legomsky (2007) recourt à un concept particulièrement stimulant, celui d'incorporation asymétrique des normes de la justice criminelle. Le concept met clairement l'accent sur la sélection auquel recourt le droit de l'immigration pour ne retenir que certains aspects punitifs du système de justice criminelle, et ce, sans incorporer les garanties juridiques et procédurales correspondantes. Mais l'expression conceptuelle choisie par Legomsky prête également à confusion. La notion d'incorporation présuppose en effet que la justice criminelle est le seul système normatif capable d'imposer une punition, et que ce sont donc ses logiques d'intervention qui contamineraient d'autres systèmes normatifs. Cet «argument de la contamination» est historiquement contestable. Le recours au droit administratif comme forme de contrôle social punitif non criminel et quasi légal n'est pas nouveau ${ }^{6}$, même s'il est sûrement devenu

4. C'était d'ailleurs un des enjeux soulevés devant la Cour suprême du Canada dans Charkaoui c. Canada (Citoyenneté et Immigration), [2007] SCC 9. En réponse, la Cour a validé le fait de recourir à des lois sur l'immigration pour assurer la sécurité nationale.

5. «Alors que les caractéristiques policières et punitives de la justice criminelle ont été importées, celles qui sont liées aux poursuites judiciaires ont été consciemment rejetées, en particulier l'ensemble des droits procéduraux habituellement reconnus en matière criminelle. Plutôt qu'évoquer la simple importation d'un modèle de justice criminelle, une observation plus fine devrait donc montrer comment le droit de l'immigration intègre progressivement les théories, les méthodes, les perceptions et les priorités propres à la dimension punitive de la mise en application des normes de la justice criminelle. Mais montrer aussi comment, parallèlement, ce droit de l'immigration rejette les procédures criminelles et ce, en faveur d'un régime réglementaire civil»(Legomsky, 2007 : 472, notre traduction).

6. Johan Steyn (2004) nous rappelle qu'en temps de crise, le recours au droit administratif, et en particulier au droit militaire, est en fait un choix récurrent à travers l'histoire. Il note par exemple que durant la seconde guerre mondiale, le recours à la détention sans inculpation ni jugement était légion. Les tribunaux qui s'en remettaient à l'exécutif pour 
plus explicite après le 11 septembre 2001, notamment avec l'usage croissant du droit militaire et des lois sur l'immigration dans le cadre de la guerre menée contre le terrorisme. En somme, tant le concept de criminalisation que celui de l'incorporation asymétrique apparaissent comme quelque peu problématiques. Dans cet article, nous pousserons donc plus loin la réflexion initiée par Legomsky, en questionnant les deux fronts du consensus autour de la criminalisation. Plus spécifiquement, nous avancerons 1) que le contrôle de l'immigration relève moins d'un processus de criminalisation que d'un processus de mise en forme administrative des litiges, et 2) que la punition imposée par les lois en matière d'immigration ne renvoie pas à des peines (poena) mais bien à des mesures de police.

De notre point de vue, le contrôle de l'immigration au Canada peut être adéquatement décrit comme un état d'exception continu et toujours sous l'autorité de la loi, comme une sorte de zone grise (Dyzenhaus, 2006; Vermeule, 2009) dans laquelle les contraintes qui pèsent sur l'action ministérielle sont si faibles qu'elles permettent au gouvernement d'agir non seulement comme bon lui semble, mais souvent aussi avec l'approbation d'un tribunal. Nous mettrons plus spécifiquement l'accent sur la composante judiciaire du contrôle de l'immigration ${ }^{7}$, en montrant comment les événements sont traduits et connaissent une mise en forme pénale spécifique à la Commission de l'immigration et du statut de réfugié (CISR), mais aussi comment ce type de punition se distingue des formes classiques de châtiments qu'on retrouve dans le droit pénal.

Nos observations sont tirées de l'analyse documentaire de différents textes de loi, réglementations et autres lignes directrices régulant la CISR. Elles se fondent également sur les décisions rendues par cette Commission ainsi que sur notre travail de terrain ethnographique visant à saisir ses pratiques (Bourdieu, 1990) et ses sensibilités juridiques (Geertz, 1986); un travail de deux ans qui a été mené dans la région est de la CISR (Montréal et Ottawa). La CISR, le tribunal administra-

prendre leurs décisions constituaient la norme. De même, quand Foucault (1995) mentionne la Loi sur les pauvres de 1834, il évoque une loi qui ne relève non pas du droit pénal mais bien du droit administratif. .

7. Tous les cas ne se rendent pas au tribunal. Les immigrants illégaux et les visiteurs relèvent par exemple de la sécurité intérieure et du maintien de l'ordre, et sont presque traités exclusivement par l'Agence des services frontaliers du Canada (ASFC), dans le cadre de leurs fonctions de police. 
tif indépendant le plus important au $\mathrm{Canada}^{8}$, est un tribunal fédéral spécialisé qui opère dans tout le pays. Cette commission est responsable à la fois de l'administration judiciaire des litiges en matière d'immigration et des décisions concernant les demandes de statut de réfugié faites au Canada. Le tribunal est composé de quatre sections: Section de la protection des réfugiés (SPR), Section d'appel des réfugiés (SAR) ${ }^{9}$, Section de l'immigration (SI) et Section d'appel de l'immigration (SAI), chacune de ces sections disposant de ses propres commissaires, lignes directrices, procédures et pratiques. Nous avons décidé d'exclure de l'analyse les cas des réfugiés et les appels concernant les demandes de parrainage non seulement pour éviter de nous disperser dans notre argumentation, mais aussi par manque d'espace. Le portrait que nous brosserons de la CISR repose, pour l'essentiel, sur les pratiques et les décisions entièrement tournées vers la punition.

Notre argumentaire sera structuré en deux axes principaux. Dans le premier, intitulé $A u$-delà des crimes, nous examinerons le style administratif de mise en forme pénale (Acosta, 1987) en jeu à la CISR, en mettant surtout l'accent sur les règles en matière de preuves, les aspects procéduraux et les enjeux symboliques clés de la SI et de la SAI. Quant au deuxième axe, $A u$-delà des peines, nous y présenterons diverses formes de punitions présentes dans le droit de l'immigration. Alors que ces punitions semblent similaires aux châtiments imposés par le droit pénal (détention, renvoi et surveillance), nous insisterons sur le fait qu'elles sont en réalité essentiellement préventives et que la plupart du temps, elles ne suivent pas les mêmes principes en termes de détermination de la peine. Ces deux axes illustrent deux aphorismes majeurs: 1) les immigrants

8. Jusqu'à récemment, la véritable indépendance de la CISR était questionnable dans la mesure où ses commissaires avaient des mandats à titre temporaire (généralement de trois ans, renouvelables). Les «motivations politiques» jouaient par ailleurs un rôle important dans les nominations et les renouvellements, comme nous l'apprendrons par d'autres chercheurs (par exemple, Crépeau et Nakache, 2008) mais aussi par différents commissaires lors de notre travail de terrain. La combinaison de ces deux variables (mandats à titre temporaire et «motivations politiques») apparaissait hautement problématique parce qu'elle plaçait les commissaires dans une position vulnérable pour garder leur emploi. Comme le dira un de nos interlocuteurs: «Si tu n'assures pas un "bon rendement" (vois-tu ce que je veux dire), tu n'auras pas de renouvellement... une amie à moi n'a eu qu'un renouvellement d'un an et elle a bien saisi le message».

9. La SAR a été créée sous la LIPR (art. 110, 111 et 171), mais elle n'est entrée en vigueur que le 15 décembre 2012. La Loi visant à protéger le système d'immigration du Canada avait alors déjà reçu la sanction royale depuis approximativement six mois. Ajoutons que cette section n'existait donc pas lors de notre travail de terrain à la CISR et que, par conséquent, elle ne sera pas discutée dans notre article. 
sont loin d'être criminalisés, et 2) la plupart du temps, les immigrants ne sont pas soumis à des peines, mais bien à des mesures de police. Nous conclurons notre article en soutenant que le contrôle de l'immigration pose un certain nombre de défis théoriques et méthodologiques pour la criminologie, notamment quand ce contrôle suggère que les formes de pénalisation qui touchent l'immigration ont leur logique propre. En ce sens, nous suivons Valverde quand elle propose d'analyser les effets $\mathrm{du}$ droit en se demandant «ce que produit un ensemble restreint de pratiques et de savoirs juridiques et "comment ils fonctionnent» plutôt que de se demander «ce qu'ils sont» (Valverde, 2003: 11; Bourdieu, 1990; Valverde, 2009). Dans le cas qui nous occupe, et indépendamment des intérêts qui ont guidé l'action du législateur et les pratiques du champ politique, nous verrons ainsi que des effets juridiques concrets de la noncriminalisation, voire de la décriminalisation, peuvent en fait apparaître davantage punitifs que la criminalisation classique.

\section{Au-delà des crimes: réflexions sur la mise en forme administrative prônée par la CISR}

Une des premières leçons qu'apprennent les étudiants dans leurs cours sur la Loi sur l'immigration et la protection des réfugiés, c'est que toute possibilité d'accès aux tribunaux est toujours préférable à une situation où on est amené à devoir seulement traiter avec la bureaucratie de l'immigration. Comme indiqué plus haut (supra note 7), une part substantielle du contrôle et de la pénalisation de l'immigration relève du simple maintien de l'ordre (policing). L'Agence de services frontaliers du Canada (ASFC) contrôle l'immigration, mais tout en ayant un mandat plus large que la police publique traditionnelle. Outre leur rôle de maintien de l'ordre et de gouvernance des étrangers, les agents de l'ASFC agissent également comme procureurs de l'État au tribunal (CISR) mais aussi comme juges parce qu'ils ont juridiction sur les résidents non permanents (pour lesquels l'ASFC n'a pas besoin de l'aval de la CISR). En d'autres mots, les immigrants illégaux et les résidents temporaires peuvent être arrêtés, détenus et/ou expulsés directement par l'ASFC et à sa discrétion. Non seulement l'Agence n'a pas à produire des crimes pour agir (Ericson, 1981), mais surtout les individus qu'elle prend en charge n'ont pas accès à un tribunal. Ajoutons que des procédures exceptionnelles telles que les certificats de sécurité, qui permettent de détenir et de déplacer des étrangers, se situent elles aussi complètement en dehors 
du champ de la CISR. Dans les paragraphes qui suivent, nous ne mettrons par conséquent l'accent que sur une facette plus limitée du contrôle de l'immigration, à savoir le type de système de justice qui prend en charge les immigrants avec un statut stable au Canada. Nous dresserons ainsi un rapide portrait de la façon dont la Section de l'immigration et la Section d'appel de l'immigration traitent les résidents permanents quand ils violent la LIPR et/ou ses règlements. Cette brève description nous aidera à clarifier la manière dont les événements se retrouvent judiciairement traduits (Acosta, 1987) dans le système d'immigration et comment ce processus de pénalisation se distingue du processus de criminalisation classique (système de justice criminelle).

La Section de l'immigration (SI) mène deux types d'audiences: enquêtes en matière d'immigration (entrée et séjour) et contrôle des motifs de détention. La première est tenue pour décider si un étranger ou un résident permanent est inadmissible à entrer au Canada et donc susceptible d'en être renvoyé. Le second type d'audience consiste en un examen des fondements de la détention. Il s'agit de décider si un étranger doit rester en détention ou pas (et s'il doit être remis en liberté, à quelles conditions). En théorie, les deux audiences sont de type accusatoire. Elles incluent un contre-interrogatoire et d'autres procédures judiciaires associées à un procès juste et équitable. Or, les basses exigences en matière de preuve et la présomption que les arguments du représentant de l'État («le conseil du ministre») sont plus crédibles que ceux avancés par l'immigrant affectent significativement l'équilibre entre les parties, minant l'équité des procédures. L'enjeu clé dans la SI est l'usage de la notion de responsabilité stricte pour évaluer la responsabilité de l'étranger dans la violation des règles à respecter en matière d'immigration. Cet état de fait structure symboliquement la SI de façon à ce qu'en pratique, il n'y ait finalement rien à débattre ni à contester. Pour illustrer nos propos, au début de notre travail de terrain, différents acteurs nous ont vivement découragés de mener toute observation à la SI, arguant du fait que ce serait une «perte de temps». Ainsi, là où les avocats des appelants nous informaient "qu'il n'y a pas grand-chose à faire [à la SI] parce que d'emblée, l'immigrant part perdant», les conseils $\mathrm{du}$ ministre soutenaient, eux, que «c'est vraiment rapide [en comparaison des procédures d'appel] (...), les commissaires de la SI ne disposant pas d'un important pouvoir de juridiction [d'une grande influence pour orienter les décisions]». Sans surprise, et comme nous le détaillerons plus loin, les décisions de la SI sont hautement portées vers l'exclusion de 
l'immigrant, favorisant à la fois le fait qu'il reste en détention et les mesures de renvoi.

La Section d'appel de l'immigration (SAI) suit le même système de procédure et les mêmes règles en matière de présentation de la preuve que la SI mais, dans cette section, étonnamment, le système accusatoire fonctionne (du moins comme il est supposé fonctionner dans un système normatif avec des exigences de preuve peu élevées). La différence se situe dans le fait que la SAI peut prendre en compte des raisons d'ordre humanitaire ${ }^{10}$. Cette possible prise en compte change complètement la dynamique de résolution des conflits dans la mesure où les motifs d'ordre humanitaire $\left(\mathrm{HC}^{11}\right)$ ouvrent la voie à de vrais débats et au contradictoire. C'est ainsi que même si techniquement, la norme de responsabilité reste la même, les intentions de l'appelant deviennent un aspect-clé quand sont prises en compte les HC. Dès qu'il y a présence de HC, l'appelant est amené à débattre sur les violations qu'il a commises par rapport à la LIPR (et/ou au Code criminel canadien). Les conditions imposées par toute autorité constituée (police, ASFC, tribunal pénal, service correctionnel, SI, etc.) sont également discutées (les conditions ont-elles été remplies?) tout comme d'autres circonstances liées au cas étudié (par exemple, l'intérêt supérieur de l'enfant).

La SAI traite différents types de conflits incluant des appels suite à un refus d'une demande de parrainage, des appels pour des individus qui n'ont pas réussi à remplir les obligations exigées en matière de résidence, des appels suite à des décisions d'enquête en matière d'immigration de la SI (appel d'une mesure de renvoi) ou encore des appels du ministre d'une décision de la SI (ces derniers appels sont très rares puisque devant la SI, le ministère ne perd que dans environ $3 \%$ des $\operatorname{cas}^{12}$ ). De façon générale, faire appel d'une décision rendue par la bureaucratie de l'immigration (demandes de parrainage et obligations de résidence) tend à être plus aisé pour les immigrants dans la mesure où les commissaires de la SAI sont relativement ouverts à envisager qu'un agent des visas ou l'AFSC ont soit commis une erreur de droit ou de fait soit n'ont pas

10. Trois critères permettent des appels (du moins en ce qui concerne les mesures de renvoi) : «(d) la décision attaquée est erronée en droit, en fait ou en droit et en fait»; «(b) il y a eu un manquement à un principe de justice naturelle»; ou «(c) (...) il y a des motifs d'ordre humanitaire justifiant, vu les autres circonstances de l'affaire, la prise de mesures spéciales» (LIPR; art. 67.1).

11. Le terme HC est couramment utilisé sur le terrain (CISR), même en français. Abréviation de son équivalent en anglais (bumanitarian and compassionate considerations).

12. CISR, 2002b, 2003, 2004, 2005, 2006, 2007, 2008, 2009a, 2010, 2011, 2012. 
observé un principe de droit naturel (LIPR, art. 67.1(a) et (b)). En revanche, se fonder sur les articles 67.1(a) et/ou (b) pour faire appel d'une décision d'enquête en matière d'immigration de la SI reste très rare parce que les questions de fait et de droit ne font presque pas l'objet de débat dans cette section de la CISR. En fin de compte, la seule option viable pour les personnes faisant appel de mesures de renvoi passe par des HC (LIPR, art. 67.1(c)) parce que dans de tels cas, il y a au moins matière à débattre et donc à contester. Ces dynamiques étaient récurrentes durant notre travail de terrain. Dans les quelques cas que nous avons observés où l'appelant estimait que la SI avait commis une erreur, la personne avait généralement renoncé à s'appuyer sur l'article 67.1(a) et/ou (b) durant la conférence préparatoire à l'audience. Elle interjetait appel sur la seule base des HC. Bref, quand on regarde plus largement les deux sections, il est intéressant de noter que ces $\mathrm{HC}$, qui relèvent d'une situation supposée exceptionnelle, constituent le seul critère à même de rétablir les principales caractéristiques des institutions juridiques (de «régler un litige»-Bohannan, 1965), mais aussi de fournir les meilleurs arguments pour éviter ou atténuer la punitivité du système d'immigration.

Les règles en matière de preuves sont au cœur de tout système normatif. Elles constituent leur fondement épistémologique : l'ensemble de procédures, de techniques et de pratiques servant à orienter la manière dont le savoir est construit ou doit être construit. Ces règles se structurent généralement autour de trois questions ou principes majeurs. Premièrement, quelles sont les données admissibles? Ensuite, à qui incombe-t-il de prouver une affirmation litigieuse, ou encore à qui revient le fardeau de la preuve? Enfin, troisièmement, quel est le niveau de vérification requis pour valider une prise de position donnée et/ou un élément d'information, ou encore quel est le niveau de la preuve? C'est l'arrangement des réponses à ces questions épistémologiques qui, ajouté aux critères de responsabilité et au système procédural en place, déterminera plus ou moins l'ontologie d'un tribunal. Si on ajoute la jurisprudence et les pratiques, nous avons les éléments qui constituent la vision du monde (weltanschauung) d'un tribunal, la manière dont ses commissaires interprètent, définissent et se représentent la réalité sociale. Ou pour reprendre les mots de Acosta: "la transformation progressive de ce qui, au départ, était une "trame de vie" en "fait juridique" (1987: 2).

Selon la LIPR, la Commission de l'immigration et du statut de réfugié (CISR) «n'est pas liée par les règles légales ou techniques de présentation de la preuve» et «peut recevoir les éléments qu'elle juge crédibles 
ou dignes de foi en l'occurrence et fonder sur eux sa décision ${ }^{13}$. En d'autres mots, la CISR peut en pratique tout recevoir. Concernant le second principe, la règle formelle veut que le fardeau de la preuve repose sur le demandeur, peu importe qu'il demande un statut de réfugié (le «réfugié»), qu'il exige une mesure de renvoi (le ministère), qu'il fasse appel en matière de parrainage (un citoyen qui parraine), etc. C'est surtout vrai pour la SI puisque, dans le cas de la SAI, le fardeau de la preuve peut se déplacer d'une partie à l'autre en raison des $\mathrm{HC}$ ou en fonction de la dynamique des résolutions de conflits. Il arrive par exemple que le conseil du ministre se retrouve clairement indisposé quand de façon flagrante, l'avocat de l'appelant s'est très mal préparé pour la procédure d'appel. Dans ce cas de figure, le conseil produira aussi des preuves pour l'appelant. À certaines occasions, il nous a même approchés lors de pauses et/ou durant l'heure du midi pour nous dire combien il «se sentait mal quand l'avocat [de l'appelant] ne faisait pas son travail». Et ajoutait par exemple: «Vous avez vu? C'était à moi de produire des preuves pour lui. Je ne suis pourtant pas ici pour faire sa job, mais bon, en même temps, ce n'est pas juste pour l'appelant d'avoir à ses côtés un avocat qui reste là, assis à ne rien faire». Parallèlement, à la fois comme technique de contre-interrogatoire et comme stratégie en matière de litiges, les avocats des appelants anticipent l'intervention du conseil du ministre en questionnant leurs propres clients. Une telle stratégie leur permet de fournir des éléments de preuve qui auraient été quoi qu'il arrive présenté par le conseil du ministre, mais qui, de cette manière, pourront peut-être jouer en faveur de leurs clients. Par exemple, en insistant sur le fait que leurs clients sont bien conscients d'avoir mal agi dans le passé et qu'ils éprouvent maintenant des remords, en laissant leurs clients contextualiser la «trame de vie» qui est à l'origine du conflit, ou encore en évoquant comment ils se sont réhabilités et les dispositions qu'ils ont prises pour éviter que de tels agissements se reproduisent à l'avenir. En somme, savoir qui a priori détient le fardeau de la preuve n'a pas vraiment d'importance; l'enjeu le plus important, c'est comment ce fardeau sera pris en charge durant les procédures.

Enfin, concernant le troisième principe, la CISR fonctionne avec la preuve telle qu'elle est présentée en matière civile et non comme en droit

13. Dans la $L I P R$, respectivement aux articles 170 (g) et (h) (dans toute affaire dont est saisie la Section de la protection des réfugiés), 173 (c) et (d) (dans toute affaire dont est saisie la Section de l'immigration), et 175 (b) et (c) (dans toute affaire dont est saisie la Section d'appel de l'immigration). 
pénal, où elle exige plus de certitude («hors de tout doute raisonnable»). Les critères généralement appliqués en termes de preuve pour l'ensemble des sections reposent sur la «prépondérance des probabilités» («il est plus probable que le contraire que...»), mais ces critères peuvent varier en fonction des types de cas entendus par la Commission. Parfois, la LIPR spécifie quelle est la présentation de la preuve recevable, sachant que la jurisprudence et les pratiques jouent elles aussi un rôle majeur. Par exemple, des exigences de preuve moins élevées telles qu'une «possibilité sérieuse» ou «des motifs raisonnables de croire» sont aussi utilisées par le tribunal. La première formulation peut ainsi être appliquée en cas de risque de torture (art. 97(1)(a)) à la SPR. Quant à la seconde, pour laquelle l'exigence de preuve est encore moins élevée, elle est généralement utilisée dans des cas de criminalité organisée (art. 37(1)(a)) à la SAI. Le critère des «motifs raisonnables de croire» est problématique, car il se situe juste au-dessus du «simple soupçon». Un tel constat est intéressant, mais surtout tragique parce que le critère le plus bas possible à être appliqué en matière d'immigration porte sur des violations semblables à des infractions criminelles; soit des cas qui exigeraient une présentation de la preuve beaucoup plus élevée si ces «trames de vie» étaient poursuivies dans le système de justice criminelle. Ce constat conduit à des situations paradoxales où la Couronne n'est pas en mesure de porter une accusation criminelle en raison de l'absence de preuves, mais où les accusations déposées par la police satisfont suffisamment les critères administratifs exigés pour pouvoir détenir des non-citoyens, pour les poursuivre par la CISR et/ou encore pour les expulser du Canada.

Un bon exemple de cette situation est la définition de membres de gangs utilisée par la Commission. Pour que le système d'immigration considère qu'un individu est membre d'un gang, il n'est pas nécessaire de disposer d'une décision émanant de la justice criminelle qui aurait préalablement établi son adhésion. Les critères établis par le Service canadien de renseignements criminels suffisent. «Utilisés par toutes les forces de police à travers le Canada» (Canada (Citoyenneté et de l'Immigration) c. Brutus, [2002]. Dossier $\mathrm{n}^{\circ}$ 0018-A2-01385), ils peuvent prendre les formes suivantes:

1) Avoir des renseignements d'une source fiable (soit membre du gang, membre d'un gang rival, source de la collectivité, autorité scolaire, commerçant, citoyens); 2) Avoir un rapport de surveillance de la police confirmant que la personne entretient des rapports avec des membres reconnus 
du gang ; 3) Aveu de la personne; 4) Participation directe ou indirecte de la personne à un crime de gang; 5) Résultats d'un procès confirmant l'adhésion de la personne à un gang; 6) Marques d'identification au gang, accomplissements de rituels initiatiques, possession d'articles et de symboles propres au gang (tatouage, armes, vêtements). (ibid.)

En somme, disposer d'un peu plus qu'un «simple soupçon» de l'implication d'un étranger dans un gang peut suffire pour l'arrêter, le détenir et/ou l'expulser. Un tel raisonnement ne se limite pas à la CISR, puisqu'il a été confirmé dans des cours supérieures comme légalement valide, notamment dans des cas de contrôles judiciaires comme Thanaratnam $c$. Canada, [2004] C.F. et [2005] C.A.F.

Monsieur Thanaratnam a été arrêté et accusé par la police plusieurs fois, mais aucune de ces accusations n'a jamais été portée devant un tribunal par la Couronne. Alors qu'une requête concernant son contrôle judiciaire avait été autorisée dans un premier temps par la Cour fédérale (renvoi devant la CISR pour une audience de novo au motif que «les conclusions de la Commission selon lesquelles Monsieur Thanaratnam était membre d'un gang ne s'appuyaient sur aucune preuve»), le gouvernement fédéral a fait appel de la décision et a gagné à la Cour d'appel fédérale un an plus tard, rétablissant la décision initiale prise par la CISR. Le raisonnement de la C.A.F. est à la fois instructif et clair concernant les critères que la Commission est en droit d'utiliser:

Le rôle de la Cour n'est pas de décider si, selon la preuve présentée à la Commission, il existait des "motifs raisonnables de croire», mais seulement de décider s'il était irrationnel de toute évidence pour la Commission de tirer cette conclusion. En l'absence d'une allégation selon laquelle la Commission a commis une erreur de droit, ou que sa procédure était inéquitable, il était difficile d'établir que la conclusion de la Commission, selon laquelle il existait des «motifs raisonnables de croire» était manifestement déraisonnable. Une conclusion n'est pas manifestement déraisonnable simplement parce que des déductions différentes de celles de la Commission peuvent être faites de façon raisonnable à partir de la preuve. Bien qu'aucun élément de preuve n'ait été déterminant dans la présente affaire, la preuve dans son ensemble était suffisante pour que la décision de la Commission ne puisse être considérée comme manifestement déraisonnable. ${ }^{14}$ (Nous soulignons)

La présomption d'innocence ne constitue pas un principe pertinent ici. Nous ne sommes pas dans une situation où la maxime in dubio pro reo 
(le doute profite à l'accusé) l'emporte sur la maxime in dubio pro rex (le doute profite au souverain). La punition administrative se fonde sur l'inversion de ces principes et donc sur la quasi-absence de garanties juridiques. Ce processus de décriminalisation punitive (Velloso, 2006) / sous-criminalisation (Ashworth et Zedner, 2010) est une voie alternative fréquemment utilisée pour punir les immigrants tout en évitant des procès pénaux. Walters (2002) rappelle d'ailleurs que ces pratiques ont cours depuis au moins le $19^{\mathrm{e}}$ siècle.

La notion de vide juridique (Steyn, 2004; Dyzenhaus, 2006; Vermeule, 2009) est très utile pour réfléchir à un tel processus et pousser un pas plus loin les arguments de Legomsky. Le concept de vide juridique a été initialement élaboré dans le contexte du droit militaire par Lord Steyn, utilisant Guantánamo Bay comme exemple. Il sera nuancé plus tard avec une distinction opérée entre les vides juridiques (trous noirs juridiques) et les zones grises (trous gris juridiques). Le concept sera par ailleurs bientôt étendu au droit administratif dans son ensemble par Dyzenhaus et Vermeule. Le second résumera les définitions du premier (Dyzenhaus, 2006) comme suit:

Les trous noirs juridiques surviennent quand la législation ou les règles juridiques vont «explicitement soit exempter le pouvoir exécutif des obligations revenant à un État de droit, soit exclure [...] tout contrôle judiciaire des actions du pouvoir exécutif en question». Les zones grises, qui sont en fait «des trous noirs déguisés», surviennent quand «il existe des contraintes juridiques qui pèsent sur l'action du pouvoir exécutif - nous ne sommes donc pas dans une zone de non-droit -, mais que ces contraintes sont si faibles qu'elles permettent au gouvernement d'agir comme bon lui semble». Les zones grises constituent ainsi «la façade ou la forme de l'État de droit et sont bien loin d'offrir aux individus des protections substantielles». (Vermeule, 2009: 1096).

Concevoir le contrôle de l'immigration et la CISR comme une zone grise permet d'être encore plus nuancé qu'avec l'idée de l'incorporation asymétrique. L'idée défendue serait celle d'un système de justice spécialisé en immigration qui est fondé sur des principes de droit administratif, mais qui n'incorpore pas nécessairement les théories ou les modes d'action de la justice criminelle. Non seulement le modèle du droit de l'immigration rejette le principe même de la poursuite criminelle, mais il ne prend pas davantage en compte les garanties juridiques les plus fondamentales. La détérioration des droits suit son cours et la CISR continue de se montrer beaucoup trop déférente à l'égard du pouvoir exécutif. 
Le contrôle judiciaire existe techniquement, mais la plupart des demandes ne dépassent même pas la phase initiale (demandes d'autorisation). Quand des cas sont finalement entendus, les Cours fédérales posent à peine des limites à l'action du pouvoir exécutif, ce qui peut être partiellement expliqué par la portée restreinte du contrôle judiciaire luimême. Il n'est en outre pas nécessaire de mobiliser l'idée schmittienne de l'exception (Schmitt, 1985) ni celle, qui l'accompagne, des libertés et des droits civils temporairement suspendus pour contrôler l'immigration puisque, par définition, les étrangers ne jouissent pas des mêmes droits ni des mêmes libertés civiles garantis aux citoyens ordinaires. Ce qui, en définitive, ressort plutôt, c'est la présence de lois, de règlements, de politiques et de pratiques somme toute fort ordinaires.

Dans le point suivant, nous discuterons des types de punitions qui peuvent être infligés aux étrangers, et montrerons ainsi comment les immigrants qui se retrouvent confrontés à un tel système sont presque toujours punis de la même façon.

\section{Au-delà des peines : réflexions sur la façon administrative de punir des conflits en matière d'immigration}

Des outils du système d'immigration peuvent être associés aux notions de punitions ou aux formes de contrôle social de type exclusif. Toutefois, elles ne sont pas tout à fait équivalentes à la notion pénale de châtiment (peine), ni même aux sanctions administratives (par exemple les amendes). Mais avant d'aller plus loin dans la réflexion sur les réponses punitives de la CISR, contextualisons brièvement la punition dans la perspective du droit de l'immigration. Dans le droit administratif, il existe deux conceptions de l'intervention juridique incluant une dimension punitive: les sanctions administratives et les mesures de police. La principale distinction entre elles tient au fait que les sanctions administratives sont considérées comme répressives et les mesures de police, préventives. Les sanctions administratives ressemblent aux peines en ce qu'elles sont toutes deux définies comme une réponse a posteriori à un événement donné (dimension répressive). Or, les garanties juridiques sont plus faibles dans le cas de ces sanctions administratives: la mens rea n'étant pas requise, la responsabilité stricte est souvent suffisante et le niveau de preuve exigé est plus bas. 
Les instruments punitifs utilisés dans le droit de l'immigration au Canada (et un peu partout en Occident) ne sont pas des sanctions administratives, mais bien des mesures de police. Elles ne constituent pas une réponse a posteriori à une infraction présumée, mais bien une sanction a priori qui a pour but de maintenir l'ordre public ${ }^{15}$. Le raisonnement juridique ne consiste pas à condamner un contrevenant, mais plutôt à prendre les mesures nécessaires pour garantir la paix sociale et l'ordre public. La logique qui revient à refuser l'entrée ou à exiger la sortie d'un étranger ne vise pas obligatoirement à infliger une réponse punitive, mais plutôt à empêcher un immigrant de mettre en danger l'intérêt national canadien et de potentiellement causer du tort dans le futur (LIPR, art. 34 à 42). Les motifs sécuritaires et sanitaires justifiant une interdiction de territoire sont très explicites en ce sens:

34. (1) Emportent interdiction de territoire pour raison de sécurité les faits suivants: a) être l'auteur d'actes d'espionnage ou se livrer à la subversion contre toute institution démocratique, au sens où cette expression s'entend au Canada; $b$ ) être l'instigateur ou l'auteur d'actes visant au renversement d'un gouvernement par la force; $c$ ) se livrer au terrorisme; d) constituer un danger pour la sécurité du Canada; $e$ ) être l'auteur de tout acte de violence susceptible de mettre en danger la vie ou la sécurité d'autrui au Canada; $f$ ) être membre d'une organisation dont il y a des motifs raisonnables de croire qu'elle est, a été ou sera l'auteur d'un acte visé aux alinéas a), b) ou c).» et «38. (1) Emporte, sauf pour le résident permanent, interdiction de territoire pour motifs sanitaires l'état de santé de l'étranger constituant vraisemblablement un danger pour la santé ou la sécurité publiques ou risquant d'entraîner un fardeau excessif pour les services sociaux ou de santé. (Nous soulignons)

Ce n'est pas une coïncidence que l'un des plus grands centres de détention d'immigrants, situé à Laval (QC), se soit appelé Centre de prévention de l'immigration. Cette situation fait clairement écho à la perspective du droit administratif et renvoie aux arguments déjà présentés lors du point précédent. Après tout, et comme Walters le montre, nous sommes confrontés aujourd'hui à des formes de régulation prélibérales qui ne sont pas sans rappeler «l'expulsion des pauvres à laquelle avait déjà recours la police locale dans l'Europe des $16^{\mathrm{e}}$ et $17^{\mathrm{e}}$ siècles» (2002: 281), à une époque où droit pénal et droit administratif étaient encore difficiles à distinguer l'un de l'autre tant ils étaient enchevêtrés à la fois dans le

15. Paolo Napoli (2011) lie les origines des mesures de police au développement des bureaucraties étatiques durant l'Ancien Régime. Ces mesures se seraient ainsi fondées sur une rationalité différente des formes contemporaines libérales du droit pénal. 
droit municipal et dans la justice royale. «Si le $20^{e}$ siècle témoigne d'une juridicisation de l'expulsion dans le droit national et international», l'auteur ajoute que «dans sa conception initiale, on parle bien d'une mesure administrative et non d'une mesure juridique» (Walters, 2002: 281). Au même titre que le concept de vides juridiques, ce point représente un deuxième contre-argument à opposer au point de vue de Legomsky: le pouvoir de police prélibéral (très peu encadré) comme les mesures de police ont des fondements administratifs. L'usage punitif du droit administratif n'est donc pas nécessairement la conséquence d'une extension ou «d'un élargissement de l'archipel carcéral», comme il est parfois suggéré (Cohen, 1985; Feeley et Simon, 1992). En fait, si un quelconque «argument de contamination » peut être avancé, ce serait plutôt l'argument inverse, à savoir des stratégies juridiques de type administratif qui ont influencé le système de justice criminelle (citons par exemple les ordonnances de "quadrilatères», la détention ou les ordonnances préventives, les périmètres de sécurité). Comme nous le verrons plus bas, le recours à des mesures de police dans le contrôle de l'immigration est orienté vers l'exclusion et peut être imposé concurremment avec d'autres formes de punitions de différents systèmes normatifs.

\section{Détention}

Les étrangers ne sont pas incarcérés dans des centres de détention pour immigrants (appelés aussi centres de prévention) à titre de sanction criminelle. Ils ne purgent pas une peine de prison. Ils ne suivent pas un programme carcéral. Ils attendent simplement d'être remis en liberté ou encore qu'une autre mesure de police soit exécutée (par exemple, un renvoi ou une libération conditionnelle $\mathrm{e}^{16}$ ), une attente qui dépasse rarement deux mois ${ }^{17}$. Comme évoquée dans le point précédent, la Section de l'immigration contrôle les motifs de détention en appliquant des niveaux de preuve très peu élevés ainsi que les critères de la responsabilité stricte. Les conséquences des contrôles des motifs de la détention

16. Nous parlons ici de la libération conditionnelle en matière d'immigration, et non en matière de justice criminelle. Notons cependant que ces libérations et les conditions qui les accompagnent peuvent se superposer.

17. La récente affaire du $M V$ Sun Sea fait clairement figure d'exception: 443 des 492 Tamouls introduits clandestinement au Canada par le Sun Sea ont été incarcérés, coûtant plus de 22 millions de dollars pour leur détention et près d'un million de dollars pour les procédures menées par la SI (Nakache, 2011 : 58-62). 
sont assez impressionnantes en termes d'exclusion sociale. Il est tout aussi étonnant de voir à quel point les immigrants qui doivent passer par la CISR en sortent rarement indemnes. Les statistiques les plus récentes (2011-2012) montrent en effet que pour près de 18000 contrôles des motifs de détention examinés ${ }^{18}, 81 \%$ des immigrants sont restés en détention (le pic post-LIPR), $12 \%$ ont été mis en liberté sous certaines conditions, $1 \%$ ont eu leurs conditions modifiées et seulement $6 \%$ ont été libérés sans condition (CISR, 2012). Ces nombres étaient similaires en 2010-2011 (CISR, 2011 : 17-19), si ce n'est que la SI excluait encore davantage durant les années précédentes: $4 \%$ avaient été libérés sans condition en 2009-2010, $6 \%$ en 2008-2009, $5 \%$ en 2007-2008, $4 \%$ en 2005-2007, et seulement $3 \%$ en 2004-2005 (CISR, 2008, 2010). Répétons que ces modalités à remplir pour l'administration de l'immigration ne sont pas nécessairement les mêmes que celles imposées par un tribunal pénal ou par une commission de libération conditionnelle. Nous reviendrons plus loin sur cet enjeu.

\section{Mesures de renvoi}

La Section de l'immigration mène aussi des enquêtes pour déterminer si un étranger devrait rester au Canada ou quitter le pays. Comme la population et les enjeux-clés de la Section sont plus ou moins les mêmes que dans les statistiques précédentes, les résultats des enquêtes ne sont pas différents. En 2011-2012, 71 \% des 2900 enquêtes (un nombre approximatif) ont ainsi «donné lieu à la prise d'une mesure de renvoi pour constat d'interdiction de territoire»; « $4 \%$ ont donné lieu à l'autorisation d'entrer ou de demeurer au Canada»; «7\% ont fait l'objet d'un retrait de l'allégation d'interdiction de territoire par l'ASFC à l'audience»; enfin, « $16 \%$ des dossiers ont été fermés parce que les personnes ont omis de se présenter à l'audience» (CISR, 2012). Ces chiffres restent relativement stables depuis que la LIPR est entrée en vigueur.

Trois facteurs devraient être pris en considération pour mieux comprendre pourquoi ces statistiques indiquent autant de mesures de renvoi. Tout d'abord, le très petit nombre d'enquêtes en matière d'immigration (entrée et séjour) par rapport au nombre de contrôles des motifs de la

18. Notons qu'un même individu peut subir à plusieurs reprises le processus visant à contrôler les motifs de sa détention dans la mesure où ces contrôles sont assurés d'abord endéans les 2 jours, puis endéans les 7 jours et enfin tous les 30 jours (si la détention est maintenue). Le nombre réel d'immigrants en détention est en fait assez bas. 
détention peut s'expliquer par le fait qu'il y a habituellement plus qu'un contrôle par individu (supra note 18) et parce que l'ASFC peut directement émettre des mesures de renvoi si la personne n'est pas un résident permanent (résidents temporaires, visiteurs et immigrants illégaux) ou encore pour des «violations moins compliquées» (ASFC, 2004), telles que des passeports falsifiés ou de fausses déclarations. Ensuite, il faut noter que dès qu'un individu omet de comparaitre, une mesure de renvoi est émise: nous devrions par conséquent ajouter un autre $16 \%$ au nombre de renvois. Troisièmement, quand l'ASFC retire une demande de renvoi, ce retrait ne signifie pas nécessairement une permission d'entrer ou de rester au Canada; il réfère parfois à un départ volontaire. En somme, le nombre de renvois lié à des «violations plus compliquées» n'est pas de $71 \%$, son pourcentage se situe entre $87 \%$ et $96 \%$. Dans certains cas aussi, les mesures de renvoi peuvent faire l'objet d'un appel (à la Section d'appel de l'immigration) ou d'un contrôle judiciaire à la Cour fédérale. Une fois qu'elle est portée en appel, la mesure de renvoi ne sera pas exécutée jusqu'à ce qu'une décision soit rendue, mais cet état de fait ne signifie pas pour autant que l'immigrant est «libre». Une mesure de détention et/ou de surveillance peut lui être appliquée.

\section{Surveillance (modalités et sursis ${ }^{19}$ )}

Dans l'article, le concept de surveillance renvoie aux diverses formes de contrôle que Deleuze (1990 : 240) qualifie d'«échanges flottants». Ces formes de surveillance sont notamment imposées par la CISR quand elle se prononce sur des appels relatifs au contrôle des motifs de détention ou à une mesure de renvoi. Nous préférons la notion de «surveillance» à celle de «probation» dans la mesure où les conditions imposées par la CISR ne sont pas tout à fait équivalentes à celles d'une probation prononcée dans le cadre de la justice criminelle. Pour le dire autrement, les conditions de surveillance dont il est question ici ne sont pas nécessairement associées aux objectifs et aux principes de la détermination de la peine. En outre, les conditions à respecter en droit de l'immigration sont distinctes, dans la pratique, de celles imposées par des tribunaux pénaux ou des commissions de libération conditionnelle. Si on excepte les mécanismes bureaucratiques de l'ASFC (contrôle des frontières, douanes, etc.), la CISR dispose de deux formes principales de

19. Sursis dans le sens d'une révocation temporaire de la décision d'une mesure de renvoi. À ne pas confondre avec le sursis en matière de justice criminelle. 
surveillance: 1) toute modalité de surveillance associée à une remise en liberté après détention; et 2) le sursis, comme suspension temporaire, à certaines conditions strictes, d'une mesure de renvoi.

Dans les deux cas, les conditions de surveillance sont imposées à un étranger. Simplement, les modalités imposées lors de remises en liberté sont relativement légères si on les compare aux conditions qui accompagnent les sursis. Dans le premier cas, ce sont des mesures prononcées pour s'assurer que la personne comparaitra, mais aussi qu'elle se tiendra «loin des ennuis» (elle aura des comptes à rendre à ce propos à l'ASFC) et qu'elle ne troublera pas l'ordre public. Les conditions à respecter pour les sursis peuvent être plus contraignantes et sont généralement justifiées comme alternatives à l'expulsion. On peut presque tout y trouver: «obligation d'être conseillé par des prêtres», "apprendre à lire», et d'autres mesures non conventionnelles, mais aussi des welfare sanctions (par exemple, faire des efforts raisonnables pour chercher et/ou garder un emploi à plein temps) (Garland, 1981, 1985). De telles contraintes peuvent être problématiques lors de leur contrôle puisqu'en cas de manquement aux obligations convenues, l'immigrant connaîtra la détention voire l'expulsion.

Sans surprise, compte tenu des éléments qui ont déjà été avancés précédemment, les commissaires de la CISR sont libres de décider ce qu'ils veulent, du moins aussi longtemps que les décisions qu'ils prennent n'apparaissent pas «irrationnelles» ou «manifestement déraisonnables» (supra note 14). Dans le cas des conditions du sursis, la situation peut même encore «s'empirer» puisqu'il n'y a plus aucun contrôle de tribunaux supérieurs ${ }^{20}$ et apparemment plus de «zone d'atterrissage $»^{21}$ pour les sursis. Des principes de détermination de la peine aussi fondamentaux que l'uniformité, la proportionnalité, la prévisibilité et l'équité sont absents; tout est une question de discrétion. L'infraction initiale n'a pas beaucoup d'importance même si, symboliquement, elle est prise

20. «Vu que les appelants tendent à ne pas demander de contrôle judiciaire des modalités de surveillance spécifiques imposées dans le cadre d'un sursis, il n'y a aucune autorité judiciaire qui se prononce sur ces modalités dans le domaine de l'immigration» (CISR, 2002a:10-4).

21. Les juges utilisent en droit pénal le terme de «zone d'atterrissage» (ou celui de «fourchette» au Québec) pour situer la durée d'emprisonnement minimale et maximale qui peut être imposée à un criminel en comparaison d'autres cas semblables. La zone d'atterrissage est donc la zone dans laquelle un juge peut «faire atterrir» sa décision sans courir le risque de la voir renversée dans des instances d'appel (sans oublier que se retrouver dans cette zone exige aussi moins de justifications de la part du juge). 
en considération. Quiconque comparaît devant la SAI affronte le même destin, celui du bannissement. Pour l'immigrant, la situation est dramatiquement simple: soit son appel est d'emblée refusé et la mesure de renvoi est donc exécutée, soit son appel est accepté, mais là encore, les chances que cette acceptation se produise tout de suite (et donc avant que soit prononcé un sursis de la mesure de renvoi) sont très minces.

Si le commissaire de la SAI décide de geler la mesure de renvoi, il peut pour des raisons pratiques choisir la longueur du sursis entre un et cinq ans. Il prend généralement aussi en considération les modalités de surveillance qui avaient été négociées entre les parties. L'observation sur le terrain montre d'ailleurs que ces négociations jouent un rôle plus grand que les critères établis dans les Directives du CISR concernant les appels relatifs aux mesures de renvoi (2002a et 2009b: chapitres 9-10). Le commissaire peut en outre simplement «copier et coller» des modalités de surveillance qui ont été généralement imposées indépendamment de l'infraction initialement commise ou des antécédents de l'infracteur. Il peut aussi choisir des modalités spécifiques qui conviennent le mieux possible aux circonstances du cas étudié (par exemple, interdire à un individu qui a des problèmes de jeu de rentrer dans un casino ou dans un type d'établissement semblable). Par conséquent, un individu qui aurait été incarcéré un an pour la commission d'un crime peut très bien recevoir un sursis de deux ans là où un autre individu, qui aurait commis le même crime et aurait purgé une peine équivalente (lui aussi dans le système de justice criminelle), peut se retrouver avec un sursis de cinq ans. Le vieil adage juridique selon lequel «la justice dépend de ce que le juge a mangé au petit-déjeuner» paraît très plausible dans le cas de la CISR.

Le cas suivant est un bon exemple de la façon dont ces dynamiques sont totalement déconnectées à la fois du crime initial et de la peine imposée. Le conseil de l'appelant (CA) a proposé un sursis de cinq ans au conseil du ministre $(\mathrm{CM})$ afin d'arriver à un accord qui convienne aux deux parties:

$\mathrm{CA}-$ Un sursis?

CM - Pour combien de temps? [le CM était d'accord de négocier un sursis]

$\mathrm{CA}-$ Cinq ans.

$\mathrm{CM}$ - Cinq ans? [le CM était très surpris de voir le CA demander le maximum]

$\mathrm{CA}-$ Ben oui, le plus jeune des enfants aura 18 ans à la fin du sursis... 
Depuis que l'enjeu-clé pour les appels tourne autour des motifs d'ordre humanitaire, on voit - comme dans le cas ci-dessus - que c'est finalement l'intérêt supérieur de l'enfant qui est devenu le principal aspect pris en considération. Au moment du dialogue, le plus jeune enfant de l'appelant avait presque 14 ans. Il aura donc 18 ans à la fin du sursis. En somme, c'est plutôt l'âge de cet enfant qui détermine la longueur du sursis sous surveillance stricte. Ce n'est ni l'infraction ni les traits individuels de l'infracteur qui détermine la durée de la punition (à la différence du processus qui sera le plus souvent suivi dans la détermination de la peine ou devant une commission de libération conditionnelle). De façon surprenante, l'appelant a fini par recevoir un sursis de 5 ans accompagné de 16 conditions à respecter; conditions qui ont été ajoutées à celles qu'il avait déjà dans le cadre d'une probation. Les sanctions étaient à la fois consécutives et concomitantes pour au moins un an (double surveillance des services correctionnels et de l'ASFC). Par la suite, l'appelant n'a plus fait l'objet que d'un contrôle de l'immigration. Au final, le temps total de punition a été considérablement étendu pour cet appelant, passant de cinq ans (pour les peines purgées «au criminel») à environ neuf ans (sans prendre en compte les effets exponentiels des peines concomitantes).

En outre, à la fin de cette longue période punitive, il n'y a aucune garantie que l'appel soit autorisé. Le commissaire peut en effet décider de rejeter l'appel (et donc de faire exécuter la mesure de renvoi) voire d'étendre la durée du sursis, que ce soit en changeant ou en maintenant les modalités de surveillance antérieures. Il existe aussi des situations où une mesure de renvoi a été émise et où l'appel n'a pas été octroyé, mais où l'immigrant est considéré comme non passible d'expulsion (risque de torture, cas d'une personne apatride, etc.). Bref, nous sommes souvent en plein imbroglio juridique avec des modalités carcérales indéfinies. Le raisonnement est simple: si le renvoi n'est pas imposé, un autre type de mesure de police comme la détention ou la surveillance s'y substituera. C'est exactement le genre de situation qui arrive fréquemment pour les certificats de sécurité: des immigrants auxquels a été délivré ce certificat sont considérés comme inadmissibles sur le territoire, mais s'ils résistent à l'expulsion, ils sont gardés en détention ou sous des conditions de surveillance draconiennes, que ce soit pour une durée indéterminée ou jusqu'à la date de leur renvoi. Notons que ces conditions carcérales incertaines ne se limitent malheureusement pas à des cas exceptionnels. 


\section{Au-delà d'un dogmatisme criminocentrique : des défis posés à la criminologie}

Pour conclure, nous aimerions faire quelques remarques sur le cas des frères Freddy et Dany Villanueva, une histoire tragique largement rapportée par les médias québécois. Au cours de la soirée du 9 août 2008, deux policiers se rendent dans l'aire de stationnement de l'aréna HenriBourassa (Montréal-Nord). Ils questionnent des jeunes qui jouaient aux dés. Peu de temps après, une altercation éclate entre la police et les jeunes, et un policier fait feu à quatre reprises, tuant le jeune Freddy Villanueva et blessant deux autres jeunes. Le rapport du coroner n'a toujours pas été divulgué et aucune accusation n'a été portée jusqu'à présent. Notre intérêt porte surtout ici sur le frère de Freddy, Dany Villanueva, pour montrer les croisements entre crime et immigration. Au moment des faits, Dany avait un casier judiciaire (justice criminelle) et était en liberté sous caution. Une mesure de probation l'engageait à ne pas troubler l'ordre public suite à une condamnation en 2006 et à des accusations pour des événements distincts survenus en juin 2008 (vol, usage d'une arme à feu factice, violation de sa période de probation, vol à l'étalage, violation d'une ordonnance et d'un engagement annoncé une semaine plus tôt) ${ }^{22}$. Dany jouait aux dés au moment du drame et sans ces événements tragiques, il aurait probablement reçu une amende pour une infraction municipale mineure (participer à des jeux de hasard $)^{23}$. Il avait en effet violé à nouveau son ordonnance, sa probation et son engagement précédents. S'il reste difficile de cerner quand Dany a commencé à devoir composer avec le système d'immigration, on sait qu'en avril 2010, la SI a émis une mesure de renvoi contre lui et qu'en 2011, son appel à la SAI a été rejeté. La Cour fédérale a par ailleurs refusé d'entendre sa demande de révision judiciaire concernant son expulsion. Dany Villanueva a été formellement criminalisé dans le passé, mais à partir de 2008, il s'est retrouvé plongé dans une myriade de systèmes normatifs, la plupart d'entre eux de type administratif (ordonnances avant procès, liberté sous caution, probation, infractions réglementaires et en matière d'immigration).

22. R. c. Villanueva Madrid, 2011 QCCM 23266; Villanueva Madrid c. R., 2011 QCCS 4851.

23. Règlement sur les parcs, R.R.V.M., c. P-3, art. 6: «Il est interdit à quiconque visite ou fréquente un parc: [...] $10^{\circ}$ de conduire des jeux de hasard ou d'y participer». 
Le concept de criminalisation n'est pas du tout approprié pour expliquer la pénalisation des immigrants, et notamment pour comprendre des cas comme celui de Dany Villanueva. Les approches criminocentriques ne tiennent pas suffisamment compte des nuances et de la complexité de réactions sociales punitives de plus en plus polymorphiques dans nos sociétés contemporaines. Décriminaliser la criminologie n'est pas une proposition neuve ni même radicale. Elle constitue plutôt un aboutissement presque logique du paradigme de la réaction sociale. Il y a plus de 20 ans, Clifford Shearing (1989) et d'autres avaient déjà avancé une telle proposition concernant les études sur la police (policing studies). On ne peut que leur donner raison quand on voit aujourd'hui à quel point le maintien de l'ordre et la gouvernance (policing) ont changé et ne se réduisent plus aux institutions de l'État ni même à l'idée de réaction (pensons par exemple à la prévention, à la gestion des risques, etc.). Du côté des études juridiques (justice studies) en criminologie, toutefois, notre regard reste étonnamment criminocentrique, y compris quand nous approchons des sujets qui ne touchent presque pas à la justice criminelle voire qui ne sont pas administrés par elle (immigration, itinérance, criminalité des affaires, etc.).

Chercher à bâtir une société dans laquelle la diversité humaine échapperait «à un pouvoir qui criminalise» (Taylor et al., 1974: 282), c'est passer à côté des multiples facettes de la résistance offerte par la punitivité, c'est aussi mettre un peu vite des limites à ce qu'on qualifie d'intervention étatique punitive. La criminologie de demain ne pourra plus être criminocentrique, elle sera plus que probablement sommée de revoir ses propres fondations. Comme Taylor, Walton et Young l'avaient prédit: "cette "nouvelle criminologie" sera en fait une vieille criminologie, au sens où elle affrontera les mêmes problèmes que ceux qu'affrontaient déjà les chercheurs en sciences sociales classiques» (1974: 278). Cerner la réaction sociale au-delà des crimes et des peines soulève divers défis méthodologiques et théoriques pour une discipline qui a inscrit l'étude du crime dans son propre nom ${ }^{24}$. Dialoguer avec d'autres savoirs sociologiques, sociojuridiques et juridiques qui contribuent, eux aussi, à réfléchir au champ pénal est salutaire en ce sens. Bref, la nouvelle vague des études juridiques en criminologie doit maintenant s'aventurer en dehors du monde de la justice criminelle, même si c'est au prix,

24. Nous discutons plus en profondeur des implications de la punition administrative pour la criminologie dans Velloso (2013). 
pour les criminologues, de devoir partiellement abandonner leurs approches et leurs objets de recherche traditionnels. Dans le cas contraire, notre destin nous conduira vers un certain obscurantisme qui déconnectera la pensée critique de formes (étatiques) punitives aussi récurrentes que diverses et dont les effets doivent malheureusement souvent se lire en termes d'exclusion sociale.

\section{Références}

Aas, K. (2011). "Crimmigrant" bodies and bona fide travelers: Surveillance, citizenship and global governance. Theoretical Criminology, 15 (3), 331-346.

Acosta, F. (1987). De l'événement à l'infraction: le processus de mise en forme pénale. Déviance et société, 11 (1), 1-40.

Agence des services frontaliers du Canada (ASFC). (2004). Document d'information de l'ASFC 2004/03: Investigations et enquêtes d'immigration. Ottawa: ASFC.

Ashworth, A. J., \& Zedner, L. (2010). Preventive orders: A problem of undercriminalization? In R. A. Duff, L. L. Farmer, S. E. Marshall, M. M. Renzo \& V. V. Tadros (eds), The Boundaries of the Criminal Law (59-87). Oxford: OUP.

Bigo, D. (1998). Sécurité et immigration: vers une gouvernementalité de l'inquiétude? Cultures et conflits, 31-32, 13-38.

Bohannan, P. (1965). The differing realms of the law. American Antbropologist, 67 (6), 33-42.

Bosworth, M. (2008). Border control and the limits of the sovereign State. Social Legal Studies, 17 (2), 199-215.

Bosworth, M., \& Guild, M. (2008). Governing through migration control: Security and citizenship in Britain. The British Journal of Criminology, 48, 703.

Bosworth, M., \& Kaufman, E. (2011). Foreigners in a carceral age: Immigration and imprisonment in the US. Stanford law and policy review, 22, 101-127.

Bourdieu, P. (1990). The logic of practice. Stanford: SUP.

Calavita, K. (1998). Immigration, law, and marginalization: Notes from Spain. Law $\mathbb{O}$ Society Review, 32, 529-566.

Calavita, K. (2003). A «reserve army of delinquents»: The criminalization and economic punishment of immigrants in Spain. Punishment $\mathbb{G}$ Society, 5 (4), 399-413.

Cohen, S. (1985). Visions of Social Control. Cambridge: Polity Press.

Commission de l'immigration et du statut de réfugié (CISR). (2002a). Appels d'une mesure de renvoi (Directives du Services juridiques). Ottawa: CISR.

Commission de l'immigration et du statut de réfugié (CISR). (2002b). Rapport ministériel sur le rendement 2001-2002. Ottawa: CISR. 
Commission de l'immigration et du statut de réfugié (CISR). (2003). Rapport ministériel sur le rendement 2002-2003. Ottawa: CISR.

Commission de l'immigration et du statut de réfugié (CISR). (2004). Rapport ministériel sur le rendement 2003-2004. Ottawa: CISR.

Commission de l'immigration et du statut de réfugié (CISR). (2005). Rapport ministériel sur le rendement 2004-2005. Ottawa: CISR.

Commission de l'immigration et du statut de réfugié (CISR). (2006). Rapport ministériel sur le rendement 2005-2006. Ottawa: CISR.

Commission de l'immigration et du statut de réfugié (CISR). (2007). Rapport ministériel sur le rendement 2006-2007. Ottawa: CISR.

Commission de l'immigration et du statut de réfugié (CISR). (2008). Rapport ministériel sur le rendement 2007-2008. Ottawa: CISR.

Commission de l'immigration et du statut de réfugié (CISR). (2009a). Rapport ministériel sur le rendement 2008-2009. Ottawa: CISR.

Commission de l'immigration et du statut de réfugié (CISR). (2009b). Appels d'une mesure de renvoi (Directives du Services juridiques). Ottawa: CISR.

Commission de l'immigration et du statut de réfugié (CISR). (2010). Rapport ministériel sur le rendement 2009-2010. Ottawa: CISR.

Commission de l'immigration et du statut de réfugié (CISR). (2011). Rapport ministériel sur le rendement 2010-2011. Ottawa: CISR.

Commission de l'immigration et du statut de réfugié (CISR). (2012). Rapport ministériel sur le rendement 2011-2012. Ottawa: CISR.

Crépeau, F., \& Jimenez, E. (2004). Foreigners and the right to justice in the aftermath of 9/11. International Journal of Law and Psychiatry, 27 (6), 609-626.

Crépeau, F., \& Nakache, D. (2008). Critical spaces in the Canadian refugee determination system: 1989-2002. International Journal of Refugee Law, 20 (1), 50-122.

Deleuze, G. (1990). Post-scriptum: sur les sociétés de contrôle. In G. Deleuze (ed.), Pourparlers 1972-1990 (240-247). Paris: Minuit.

Dyzenhaus, D. (2006) The constitution of law: Legality in a time of emergency. New York: CUP.

Ericson, R. (1981). Making crime. Toronto: Butterworth.

Ericson, R. (2007). Crime in an Insecure World. Cambridge: Polity.

Feeley, M., \& Simon, J. (1992). New penology: Notes on the emerging strategy of corrections and its implications. Criminology, 30 (2), 449-474.

Foucault, M. (1995). Discipline and punish. New York: Vintage Books.

Garland, D. (1981). The birth of the welfare sanction. British Journal of Law and Society, 8 (1), 29-45.

Garland, D. (1985). Punishment and welfare. Brookfield: Gower.

Geertz, C. (1986). Civilisations et savoir: fait et droit en perspective comparée.

In C. Geerts (ed.), Savoir global, savoir local (207-290). Paris: PUF. 
Legomsky, S. H. (2007). A new path of immigration law: Asymmetric incorporation of Criminal Justice norms. Washington of Lee Law Review, 64, 469-528.

Mathieu, L. (2001). Double-peine: les fondements juridiques d'une discrimination légale. Mouvements, 13, 83-87.

Mathieu, L. (2006). La double peine: histoire d'une lutte inachevée. Paris, La Dispute.

Miller, T. A. (2003). Citizenship and severity: Recent immigration reforms and the new penology. Georgetown Immigration Law Journal, 17, 611-666.

Miller, T. A. (2005). Blurring the boundaries between immigration and crime control after September 11. Boston College Third World Law Journal, 25 (1), 81-124.

Nakache, D. (2011). The Human and Financial Cost of Detention of Asylum-Seekers in Canada. Consulté le 28 décembre 2012, www.unhcr.org/refworld/ docid/4fafc44c2.html.

Napoli, P. (2011). Mesure de police. Une approche historico-conceptuelle à l'âge moderne. Tracés, 20, 151-173.

Palidda, S. (1999). La criminalisation des migrants. Actes de la recherche en sciences sociales, $129(1)$, 39-49.

Pratt, A. (2005) Securing Borders: Detention and Deportation in Canada. Vancouver: UBC Press.

Sayad, A. (1998). L'immigration et la "pensée d'État": réflexions sur la "double peine". Regards Sociologiques, 16, 5-21.

Schmitt, C. (1985). Political theology. Chicago: UCP.

Shearing, C. (1989). Decriminalizing criminology: Reflections on the literal and tropological meaning of the term. Canadian Journal of Criminology, 31, 169-178.

Simon, J. (1998). Refugees in a carceral age: The rebirth of immigration prisons in the United States. Public Culture, 10 (3), 577-607.

Steyn, J. (2004). Guantánamo Bay: The legal black hole. International and Comparative Law Quarterly, 53, 1-15.

Stumpf, J. (2006). The crimmigration crisis : Immigrants, crime, and sovereign power. American University Law Review, 56 (2), 367-419.

Taylor, I., Walton, P., \& Young, J. (1974). The new criminology: For a social theory of deviance. New York: Harper \& Row.

Valverde, M. (2003). Law's dream of a common knowledge. Princeton: PUP.

Valverde, M. (2009). Jurisdiction and scale: Using law's technicalities as theoretical resources. Social $\mathcal{O}$ Legal Studies, 18 (2), 139-157.

Velloso, J. (2006). Sobre o tratamento jur'dico dado ao trabalho escravo: o movimento de descriminalização. Revista Brasileira de Ciências Criminais, 59, 90-127.

Velloso, J. (2013). Beyond criminocentric dogmatism: Mapping institutional forms of punishment in contemporary societies. Punishment $\mathfrak{G}$ Society, 15 (2). Document sous presse. 
Vermeule, A. (2009). Our Schmittian Administrative Law. Harvard Law Review, $112,1095-1149$.

Wacquant, L. (1999). Des «ennemis commodes» : étrangers et immigrés dans les prisons d'Europe. Actes de la recherche en sciences sociales, 129(1), 63-67.

Walters, W. (2002). Deportation, expulsion, and the international police of aliens. Citizenship Studies, 6(3), 265-292.

Woodward, B. (2001, October $\left.21^{\text {th }}\right)$. CIA Told To Do «Whatever Necessary» to Kill Bin Laden. Washington Post. Consulté le 28 février 2012, http://www. pulitzer.org/archives/6612.

Zedner, L. (2010). "Security, the State, and the Citizen: The Changing Architecture of Crime Control”, New Criminal Law Review, 13(2): 379-403

\section{Loi canadienne}

Loi sur l'immigration et la protection des réfugiés. L.C. 2001, c. 27.

\section{Case Law}

Canada (Citoyenneté et de l'Immigration) c. Brutus [2002]. Dossier n 0018-A201385.

R. c. Villanueva Madrid [2011] QCCM 23266

Thanaratnam c. Canada, [2004] C.F. et [2005] C.A.F.

Villanueva Madrid c. R. [2011] QCCS 4851.

ABSTRACT - This paper discusses the increasing importance of administrative punishment in the penal field, using the legal treatment given to immigration conflicts in Canada as an example. Based on documentary analysis and the results of fieldwork conducted at the Immigration and Refugee Board of Canada between 2007 and 2009, I will present some characteristics of the legal translation of conflicts in immigration law and the forms of punishment involved. I will argue that these differ substantially from those in criminal law. My ultimate goal is to question the idea of criminalization of immigration as a category capable of nuancing the complexity of administrative forms of social reaction. Instead of criminalizing immigration, I will suggest that we should understand the forms of punishment in administrative law as they are (police measures and administrative sanctions), rethinking their role in the penal field and how these forms of penalization may even supplement and/or superpose traditional criminalization processes.

KEYWORDS - Immigration Control, punishment, penology, penal Policy, Immigration and Refugee Board of Canada (IRB).

RESUMEN - El propósito de este articulo es debatir la importancia creciente de las sanciones legales administrativas en el campo penal, a partir de la judicialización de los conflictos de inmigración en Canadá. Con el aporte de la literatura y de los resultados de un estudio de campo llevado a cabo entre 2007 y 2009 en la Comisión de la inmigración y de la condición de refugiado de Canadá, presentaremos ciertas características de los conflictos en el derecho inmigratorio, así como también de su manera de punir, 
y sostendremos que éstas difieren substancialmente de aquéllas propias al funcionamiento penal. Nuestro objetivo final consistirá en problematizar la idea de criminalización de la inmigración como una categoría capaz de diferenciar la complejidad de las formas de reacción social administrativas. Sugerimos que es necesario, más bien, considerar la punición en derecho administrativo como tal (medidas policiales y sanciones administrativas) y repensar su rol en el seno del campo penal a fines de comprender mejor el conjunto de reacciones sociales en las distintas instituciones jurídico-políticas, la interacción y complementariedad de éstas, sus lógicas de gobernabilidad y de funcionamiento, asi como también sus implicaciones sociales.

PALABRAS CLAVE - Control de la inmigración, punición, penología, política penal, Comisión de la Inmigración y de la condición de refugiado de Canadá (CIRS). 